Höhere Algebra. By Helmut Hasse. Volume 2: Gleichungen höheren Grades. (Sammlung Göschen.) Berlin, de Gruyter. 1927, 160 pp.

This second part of Hasse's algebra is still more interesting than the first. The contents are apparently the same as in most textbooks on higher algebra: the theory of general algebraic equations, Galois group theory, leading up to the condition for a solution by radicals.

This algebra is however no textbook in the common sense of the word, but a modern scientific review of the fundamentals of algebra, in particular the Galois fields, wherein the last traces of the theory of functions in algebra are eliminated.

The definition of the abstract rings and fields and the fundamental properties of them were already given in vol. I. A feature of the present volume is the introduction of the ideas of Steinitz developed in his famous paper Algebraische Theorie der Körper (Journal für Mathematik, vol. 137). Steinitz's classification of the abstract fields, fields of characteristic 0 and fields of characteristic $p$, is introduced : to the last class belong for instance all finite fields, and all fields having a finite subfield. All fields containing the rational field have the characteristic 0 . By construction of successive adjunction fields a Galois field is obtained, and the main theorems on the connection between groups and equations are proved for all perfect (vollkommen) fields, that is, all fields with characteristic 0 and fieds with characteristic $p$ having certain properties. 1

It is difficult to give a satisfactory account of this excellent little book in a few lines, but it can be warmly recommended to all mathematicians interested in algebra.

\title{
Oystein Ore
}

Compléments de Géométrie Moderne. By Charles Michel. Paris, Vuibert, 1926. 317 pp. Price (unbound) 35 frs.

The name Modern Geometry is used by different writers to cover a vast and varied body of doctrine, from, say, the Simson line and up to the Lie theory, or anything else. What may be meant by "compléments" is still less certain. The preface of the book might be expected to throw some light upon the vague and unassuming title, but the book has no preface. Thus reduced to use the publisher's announcement as a substitute, one soon becomes convinced that a more ambitious caption would come much nearer to be descriptive of the contents of the work. For the author presupposes on the part of the reader a familiarity with the more elementary projective properties of conics, quadrics, and algebraic curves and surfaces in general, with the use of imaginaries in geometry, etc. In other words, he expects his reader to have mastered the advanced parts of a French Traite de Géométrie, like Hadamard's, or Rouché et Comberousse's, and in addition a book like E. Duporcq's Premiers Principes de Géométrie Moderne.

With this much as a background the author treats pencils and nets of conics and quadrics, the cubic curve both in the plane and in space, Steiner's surfaces. The method of presentation throughout the book is almost exclusively synthetic, except for the first chapter, where the author 
accords "au calcul sa part inévitable." That the author thus expedites matters is quite certain, but to say that the analytical attack and the implied principle of continuity are unavoidable is to forget the brilliant and fruitful labors of von Staudt and his successors.

The author has made a very judicious choice in the vast material available and has produced a book which duplicates no other single book in the existing literature. The book is written in the good traditional style of French clarity and is free of excessive condensation. The reader is further helped by the figures which appear when and where they are most needed. Bibliographical references are few in number, there is no index, and the table of contents is brief and summary.

We have quite a few books which may be used as a basis for a first course in synthetic projective geometry, particularly in English. The situation changes radically when it comes to a second course in this subject. M. Michel's book may be used to great advantage for such a purpose in our American schools, and the set of nearly one hundred exercises at the end of the book may be quite helpful in this connection.

\section{Nathan Altshiller-Court}

Linienspektren und periodisches System der Elemente. By Friedrich Hund.

Volume IV of the series Struktur der Materie, edited by M. Born and J. Franck. Berlin, Springer, 1927. vi +221 pp.

In this book a very successful attempt is made to give a recapitulation and a uniform, systematic presentation of the results obtained in the unravelling and classification of line spectra from the pioneer work of Bohr through the investigations by Catalán, Russell, Saunders, Pauli, Heisenberg, Schrödinger, and others. In order to present only one kind of problem to the reader at a time the author has intentionally made very little explicit use of the new quantum mechanics. Nevertheless, he makes a special point of calling attention to the difficulties and limitations inherent in the correspondence principle. Hence, from different points of view the book contains material which should appeal to the astronomer and the chemist as well as to the theoretical physicist and the spectroscopist.

Not only is the presentation relatively non-mathematical, in the sense that advanced analysis has been replaced by a vectorial algorithm devised by the author, but it is also unusually clear and logical. By commencing with the rough and then proceeding to the finer properties of spectra, the atomic model so-called is refined step by step until it becomes possible to give at least a qualitative account of all the details of the most complex spectra. By no means the least valuable feature of the book consists in the numerous, excellent tables which constitute the best and most extensive classification of spectral terms and relationships extant. It is thus evident that the text is an unusually valuable contribution to the field of line spectra.

H. S. UHLER 